\title{
Diagnosis scheme for topological degeneracies crossing high-symmetry lines
}

\author{
Tiantian Zhang $\odot,{ }^{1,2,3, *}$ Ling Lu, ${ }^{1,4}$ Shuichi Murakami $\odot,{ }^{2,3}$ Zhong Fang, ${ }^{1,5}$ Hongming Weng $\odot,{ }^{1,5, \dagger}$ and Chen Fang ${ }^{1,6, *}$ \\ ${ }^{1}$ Institute of Physics, Chinese Academy of Sciences/Beijing National Laboratory for Condensed Matter Physics, Beijing 100190, China \\ ${ }^{2}$ Department of Physics, Tokyo Institute of Technology, Ookayama, Meguro-ku, Tokyo 152-8551, Japan \\ ${ }^{3}$ Tokodai Institute for Element Strategy, Tokyo Institute of Technology, Nagatsuta, Midori-ku, Yokohama, Kanagawa 226-8503, Japan \\ ${ }^{4}$ Songshan Lake Materials Laboratory, Dongguan, Guangdong 523808, China \\ ${ }^{5}$ Collaborative Innovation Center of Quantum Matter, Beijing 100084, China \\ ${ }^{6}$ CAS Center for Excellence in Topological Quantum Computation, Beijing 100190, China
}

(Received 24 November 2019; accepted 3 June 2020; published 23 June 2020)

\begin{abstract}
Theories of symmetry-based indicators and topological quantum chemistry, while powerful in diagnosing gapped topological materials, cannot be directly applied to diagnosing band degeneracies at/between highsymmetry momenta due to the violation of the compatibility conditions. However, the only information that compatibility condition can tell us is whether there are band degeneracies at/between high-symmetry momenta or not. Here we design a recursive protocol that utilizes indicators of maximal subgroups to infer the topological information of band degeneracies crossing high-symmetry lines without implementing a heavy numerical calculation, such as the existence, type, number, configuration for the band degeneracies, and so on. For demonstration, the method is used to predict the existence of ideal Weyl phonons in $\mathrm{In}_{2} \mathrm{Te}$ and node-cage phonons in $\mathrm{ZrSiO}$, respectively.
\end{abstract}

DOI: 10.1103/PhysRevResearch.2.022066

Introduction. Theories of symmetry-based indicators [1] and topological quantum chemistry [2] are useful in diagnosing topological materials, such as topological insulators [3,4], topological crystalline insulators [5], and topological semimetals [6]. However, the application of those theories needs to meet one condition, i.e., compatibility condition, which restricts the number of each irreducible representation for the occupied band at several high-symmetry points (HSPs) in the Brillouin zone (BZ). When the condition is satisfied, there will be no band crossings at/between HSPs, as shown in Fig. 1(a), or band crossings along high-symmetry lines (HSLs) can be gapped without changing the order of bands between the occupied bands and unoccupied bands at HSPs (see the Supplemental Material for details [7]). Among the cataloged topological materials, topological semimetals diagnosed by the compatibility condition are the majority, which is more than $62 \%$ in the spinful case and $98 \%$ in the "spinless" case (ignoring spin-orbit coupling) [8-10], corresponding to the case in Fig. 1(b). For such huge amounts of topological semimetals, the only information that the previous work tells us is whether the topological degeneracy is at a HSP or along a HSL. Other topological information, such as the type, number, configuration, and positions for the topological degeneracies

\footnotetext{
*zhang.t.ac@m.titech.ac.jp

†hmweng@iphy.ac.cn

${ }^{\ddagger}$ cfang@iphy.ac.cn
}

Published by the American Physical Society under the terms of the Creative Commons Attribution 4.0 International license. Further distribution of this work must maintain attribution to the author(s) and the published article's title, journal citation, and DOI. are missed. The incomplete information for the topological degeneracies is due to the imperfect algorithm in the previous work. Therefore, a method to diagnose whole information for such topological degeneracies is highly deserved.

The importance of such information, however, is highlighted in the research of topological semimetals, such as Weyl semimetals [11-15], Dirac semimetals [16-19], and node-line semimetals [20,21]. The nontrivial topology of band degeneracies in some of these materials leads to unique surface states having "Fermi arcs" [22-25] as well as an anomalous bulk transport phenomenon known as the "quantum anomaly" [26-30]. Topologically nontrivial band degeneracies appear not only in electronic bands but are also predicted and observed in the bands of bosons such as photons [25,31-33], phonons [34-48], and magnons [49,50].

In this Rapid Communication, we develop a routine for topological-diagnosing band degeneracies located along highsymmetry lines in the BZ, which can be used in both spinful and spinless systems with/without time-reversal symmetry. In the following, we will discuss our algorithm in AI class systems as an example, which corresponds to orthogonal Hamiltonians [51-53]. (Discussions on spinful systems are in the Supplemental Material [7].) Given a band structure that violates the compatibility conditions of some space group $G$, we first identify, through a "tree-search" process, the maximum subgroup $H \subset G$, such that the compatibility conditions of $H$ are satisfied, and then compute the symmetry-based indicators with respect to $H$. The value of the symmetry-based indicator for this subgroup then reveals partial information on topological invariants of the band degeneracies protected by $G$, which has not been discussed in detail before $[6,9,10]$. For example, the lowest six phonon bands of $\mathrm{In}_{2} \mathrm{Te}$ violate the compatibility conditions of \#216. Figure 1(c) shows all 


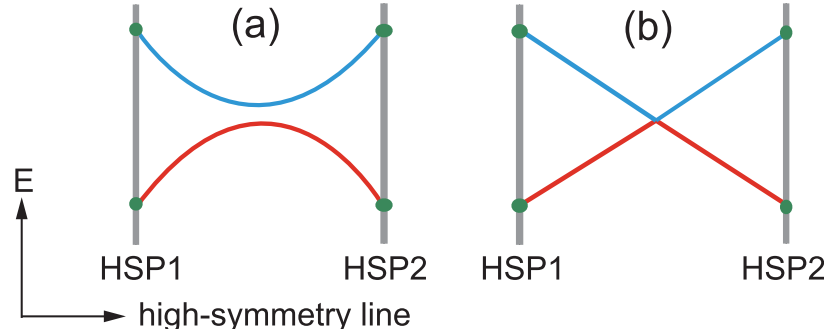

(c)

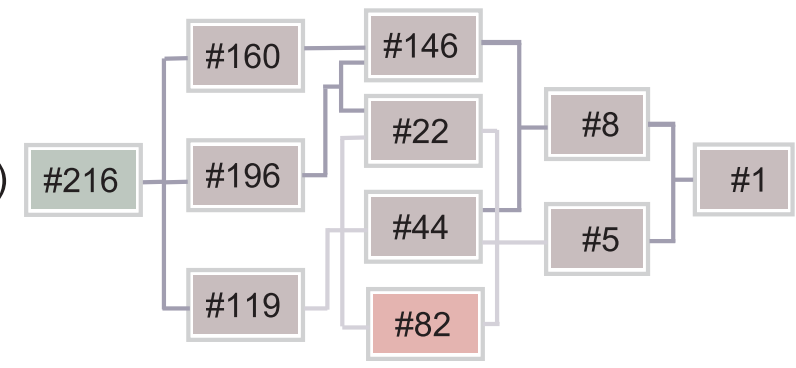

FIG. 1. (a) and (b) show two different kinds of band structure along a high-symmetry line. The red line represents the occupied band, and the blue line represents the unoccupied band. HSP1 and HSP2 represent two different high-symmetry points in the Brillouin zone. The band structure in (a) satisfies the compatibility condition, while (b) does not. (c) shows the tree-search process for \#216, which is the key step in our diagnosis algorithm. \#82 is the only maximum subgroup which has a nontrivial symmetry-based indicator and satisfies the compatibility condition for the lowest six phonon bands of $\mathrm{In}_{2} \mathrm{Te}$.

subgroups of space group \#216, from which we identify \#82 as the maximum subgroup that has nontrivial indicator group $\left(\mathbb{Z}_{2}\right)$ and satisfies the compatibility condition at the same time. We then compute the $\mathbb{Z}_{2}$ indicator for $\# 82$ and find $z_{2}=1$, which by Ref. [6] ensures that the band degeneracies between the sixth and the seventh bands are Weyl points of equal energy. We also apply the method to the phonon bands of $\mathrm{ZrSiO}$ and show the presence of a "nodal cage" in its band structure.

Flowchart for the recursive algorithm. The diagnosing process for topological degeneracies in the Brillouin zone is summarized in Fig. 2(a), which consists of five steps. In step I, symmetry data, i.e., irreducible representation, at a given list of high-symmetry momenta should be calculated by firstprinciples calculations. In step II, analyze if two conditions, i.e., the nontrivial-symmetry-based-indicator-group condition (INDC) and compatibility condition (CC) are satisfied by the space group and the symmetry data, respectively. If INDC and CC are both satisfied, which corresponds to "YY" in the flowchart, then we can calculate the symmetry-based indicator directly by Ref. [6] to get the information of topological band degeneracies at generic momenta in the BZ. If INDC is not satisfied while $\mathrm{CC}$ is, which corresponds to "NY" in the flowchart, then the system is in a "trivial" state. (Here "trivial" means that of an atomic insulator, i.e., band degeneracies in the Brillouin zone, can be gapped out without changing the order of bands between the occupied bands and unoccupied bands at high-symmetry momenta.) In other cases, CC is not satisfied, i.e., band degeneracies will exist at high-symmetry points or along high-symmetry lines, so we should use a new method to diagnose the information for band crossings in the BZ. In step III, we find all the maximal subgroups of $G$, and choose one subgroup $H$ from this set, and test if the INDC and $\mathrm{CC}$ are satisfied: If the answer is YY, we proceed to step IV; if the answer is others, we replace $H$ by its lower subgroups to repeat the recursive process step III, until we get an answer of YY or the subgroup is \#1. In step IV, we should use the symmetry-based indicator formula of $H$ to diagnose the topological degeneracy information of space group G, no matter whether the indicator is zero or not. In step V, the indicator of the subgroup $H$ can tell us the information of topological degeneracies crossing high-symmetry lines, such as types, configuration, positions, and topological charge for the topological degeneracies in the BZ. If the process stops at \#1 in step IV, we conclude that the nodes cannot be further diagnosed by eigenvalues at all (nondiagnosable). After the process, each band node protected by $G$ is to be found either as nondiagnosable, or as having a list of subgroups with corresponding indicators, which carry topological information on these nodes.

Figure 2 also lists two materials for the demonstration of our recursive algorithm in phononic systems. Figure 2(b) is an example of $\mathrm{In}_{2} \mathrm{Te}$, which has a noncentrosymmetric structure and 12 ideal Weyl points in the phonon spectra. Figure 2(c) is an example of $\mathrm{ZrSiO}$, which has a centrosymmetric structure and nodal cage band degeneracies in the phonon spectrum. Both of the cases break CC in step II.

Diagnosing process for ideal Weyl phonons in $\mathrm{In}_{2} \mathrm{Te} . \mathrm{In}_{2} \mathrm{Te}$ belongs to a $\mathcal{P}$-broken space group $F \overline{4} 3 m$ (\#216) [54], as shown in Fig. 3(a). The band crossing at around $3.22 \mathrm{THz}$ $\left(E_{w}\right)$ indicates that $\mathrm{CC}$ is broken along the $X-W$ direction for the lowest six bands, as shown in Fig. 3(e). In the following, we will get the complete information for the band crossing at around $3.22 \mathrm{THz}$ by using the diagnosing method shown in Fig. 2(a).

After obtaining symmetry data for \#216 in step I $[55,56]$, we notice that CC for \#216 is not satisfied along the $X-W$ direction, which is not a surprise because of the band crossing in phonon spectra. However, there is not a nontrivial indicator for space group \#216 in step II. Therefore in step III, we

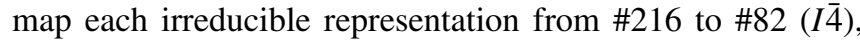
which is the maximum subgroup both having a nontrivial indicator $\mathbb{Z}_{2}$ and satisfying CC. In step IV, we calculate the topological invariant of subgroup \#82 and get a nonzero indicator $z_{2}=1$, which indicates that there will be $4 \bmod 8$ Weyl points on the $k_{z}=0$ plane (which is also the $k_{3}=0$ plane) between the sixth and seventh bands. In the following, we will provide an intuitive perspective to understand how to get the complete information for the topological degeneracies at high-symmetry momenta for \#216 from the indicator of the subgroup \#82.

Figure 3(c) shows the BZ for space group \#82, which has a similar shape with the one for \#216 shown in Fig. 3(b). Since there are two pieces of plane for the $k_{3}=0$ plane in Fig. 3(c), we can rebuild the BZ by dividing the blue quadrilateral plane into four pieces and fill them into the pink plane separately to get a new quadrilateral $k_{3}=0$ plane. In this case, the highsymmetry point $M_{1}$ in the old BZ is $M_{2}$ in the new BZ. Since there are $4 \bmod 8$ Weyl points on the $k_{1}=0$ plane, shown by red and green dots in Fig. 3(c), we find that there will be 


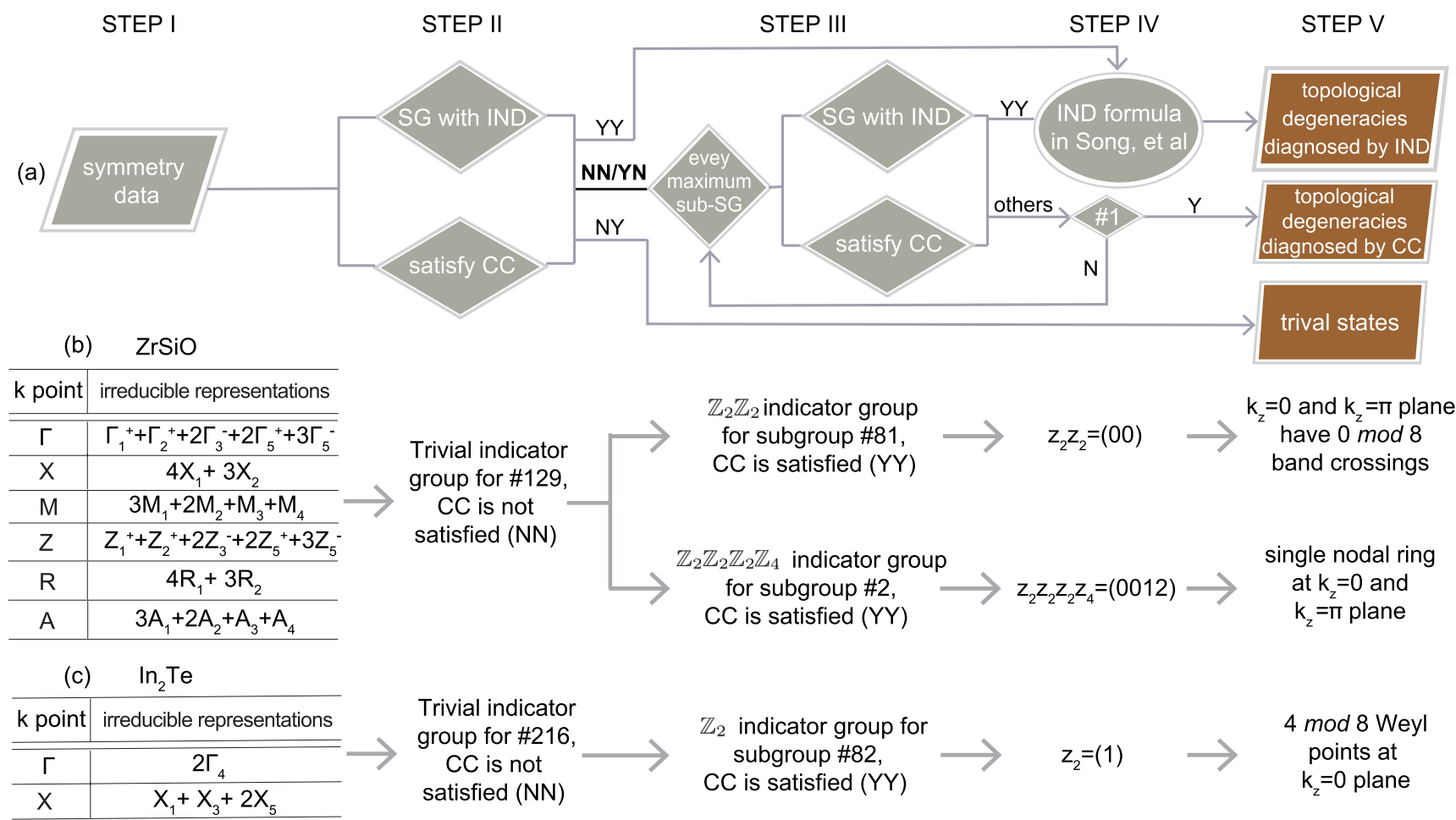

FIG. 2. (a) Calculation steps for diagnosing topological degeneracies in the Brillouin zone. SG: space group; IND: indicator; CC: compatibility condition; N: No; Y: Yes. Firstly, obtain the symmetry data by first-principles calculations, and feed them into the next step. Secondly, check whether the space group $G$ has a nontrivial indicator and whether the symmetry data satisfies compatibility conditions. If both the answers are yes, we can use the symmetry data to calculate the indicator directly and get the information of topological degeneracies at generic momenta for AI class systems. If the answers are "NY," which corresponds to the condition of "No" to "space group with nontrivial symmetry-based indicator" and "Yes" to "satisfy compatibility condition," the material is a topologically trivial one, i.e., no topological degeneracies exist in the Brillouin zone. Otherwise, topological degeneracies will exist at high-symmetry momenta, such as high-symmetry points and high-symmetry lines. Thirdly, we should find all the maximum subgroups $H$ which have a nontrivial symmetry-based indicator group and satisfy the compatibility condition at the same time in the tree-search process. After calculating the symmetry-based indicator for each maximum subgroup $H$ in step IV, we can get information of topological degeneracies for space group $G$. However, if we cannot find a subgroup $H$ in step III, then the topological degeneracies only can be diagnosed by compatibility condition. (b) $\mathrm{In}_{2} \mathrm{Te}$ and (c) $\mathrm{ZrSiO}$ are two examples for demonstrating our diagnosis scheme for AI class systems.

12 mod 24 Weyl points in the $\mathrm{BZ}$ after considering all the symmetry operators of \#216, especially $C_{3}$ symmetry around the (111) direction. A detailed calculation confirms that there are 12 robust Weyl points related to each other by symmetries at around $3.22 \mathrm{THz}$, confined on the $k_{1}=0, k_{2}=0$, and $k_{3}=$ 0 planes.

We note that 12 Weyl phonons at around $3.22 \mathrm{THz}$ are ideal ones, which are related to each other by symmetries and have equal energies, as shown in Fig. 3(d). There are another 12 Weyl phonons at around $2.58 \mathrm{THz}$, with the same indicator $z_{2}=1$ for the subgroup \#82. Surface states and surface arcs for Weyl phonons are discussed in the Supplemental Material [7].

Diagnosis process for $\mathrm{ZrSiO}$. $\mathrm{ZrSiO}$ has a centrosymmetric structure with space group P4/nmm (\#129) [57], as shown in Fig. 4(a). $\mathrm{ZrSiO}$ is a nodal-line semimetal for electronic structures when the spin-orbit coupling is ignored [20] and features the same topological properties for phonon spectra. Figure 4(b) shows the phonon bands for $\mathrm{ZrSiO}$, which have several band crossings at around $13 \mathrm{THz}$ along several highsymmetry lines. We will discuss what they are, and whether they are topologically protected in the following.
After obtaining the symmetry data for phonons by densityfunction perturbation theory in step I, we find out that they do not satisfy the $C C$ along $\Gamma-X, \Gamma-M, Z-A$, and $Z-R$ directions for the 14 lowest bands in step II. Violation of CC indicates that a band degeneracy will exist between the 14th and 15th bands at those four high-symmetry lines, which also means that we can use the recursive algorithm to figure out the complete topological information for the degeneracies. In step III, we find that the maximum subgroups satisfying INDC and $\mathrm{CC}$ are \#81 and \#2 after iterating several times. Here, we would like to note that \#81 and \#2 belong to different "tree branches," which means that neither of them is a subgroup of the other one.

The indicator group for \#81 is $\mathbb{Z}_{2} \times \mathbb{Z}_{2}$, and the corresponding topological invariants for phonons in $\mathrm{ZrSiO}$ are (00). Even though the topological invariants are zero, they can still tell us that there will be $0 \bmod 8$ band crossings at both the $k_{z}=0$ plane and the $k_{z}=\pi$ plane. As shown in Fig. 4(d), band crossings are marked by pentagrams. Since $(\mathcal{P} \mathcal{T})^{2}=+1$ in $\mathrm{ZrSiO}$, all the band crossings must belong to nodal lines/rings. Therefore a possible configuration for the band degeneracies in $\mathrm{ZrSiO}$ diagnosed by $\# 81$ is $0 \mathrm{mod}$ 
(a)

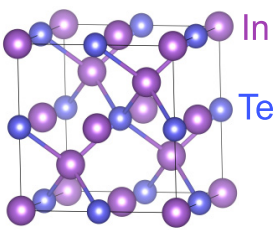

(b)
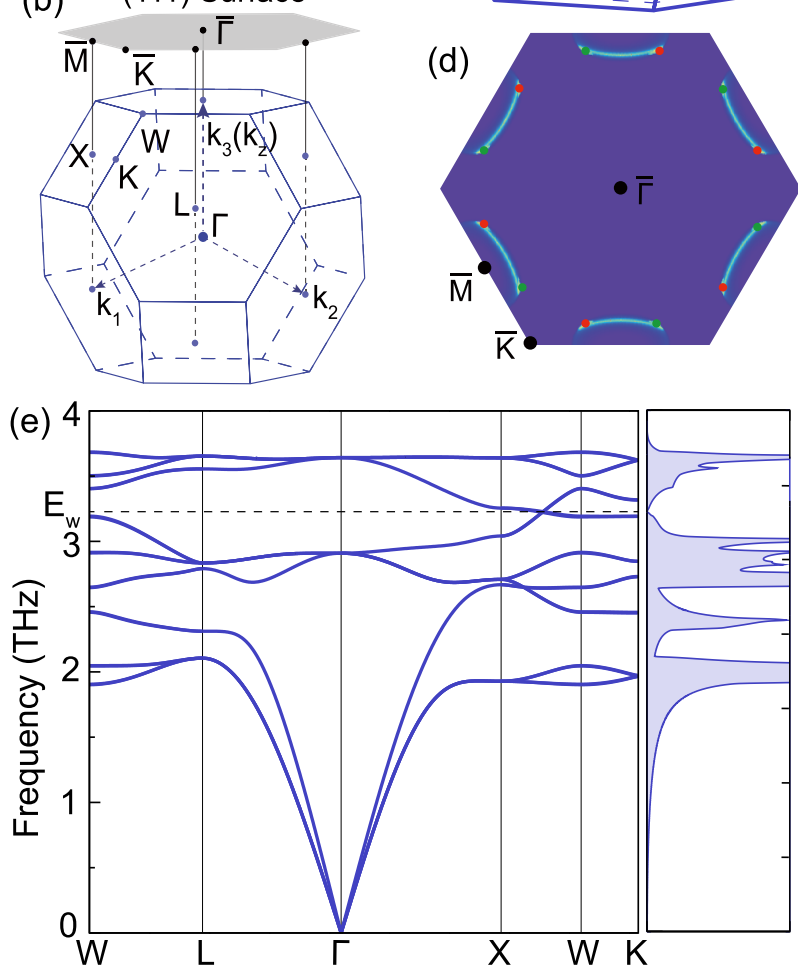

FIG. 3. (a) Crystal structure for $\operatorname{In}_{2}$ Te. (b) Brillouin zone and surface Brillouin zone along the (11) $)$ direction for \#216. (c) Brillouin zone for \#82. (d) Distribution for 12 ideal Weyl points between the sixth and seventh phonon bands in the surface Brillouin zone at $E_{w}$, where green dots represent Weyl points with Chern number of -1 and red dots represent Weyl points with Chern number of +1 . (e) Phonon spectra for $\operatorname{In}_{2} \mathrm{Te}$.

8 nodal lines crossing both the $k_{z}=0$ plane and the $k_{z}=\pi$ plane along the (001) direction, as shown in Fig. 4(d).

The indicator group for $\# 2$ is $\mathbb{Z}_{2} \times \mathbb{Z}_{2} \times \mathbb{Z}_{2} \times \mathbb{Z}_{4}$, and the corresponding topological invariants are (0012) for phonons in $\mathrm{ZrSiO}$. Those nonzero indicators tell us another possible configuration for the topological degeneracies in BZ, and we offer two different perspectives to understand it. (i) Because indicator groups are Abelian groups, indicators satisfy the sum rule. Thus (0012) can be written as $(0001)+(0011)$, which indicates a single nodal ring around the $\Gamma$ and $Z$ points by Ref. [6], respectively. $M_{z}$ symmetry in $\mathrm{ZrSiO}$ will restrict those two nodal rings in the $k_{z}=0$ and $k_{z}=\pi$ plane, which corresponds to the configuration shown in Fig. 4(e). (ii) $z_{2}=1$ and $z_{4}=2$ indicate that any curved surface in the BZ passing $\Gamma, X$, and $M$ points (or $R, A$, and $Z$ points) will be crossed by nodal lines $2 \bmod 4$ times. Therefore one possible configuration is one nodal ring lying on the $k_{z}=0$ and $k_{z}=\pi$ plane, respectively, which also matches Fig. 4(e) well.

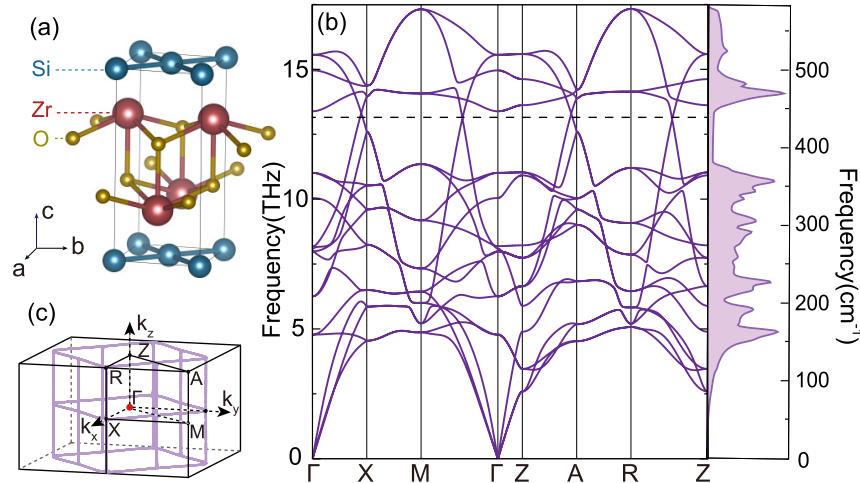

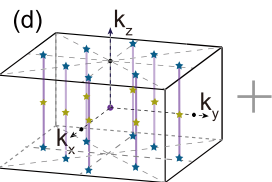

\#81

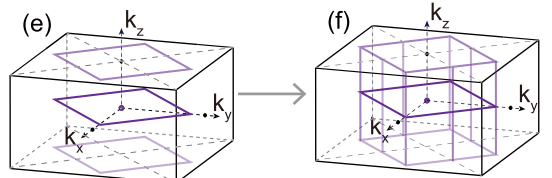

\#2

\#129
FIG. 4. (a) Crystal structure, (b) phonon spectra, and (c) Brillouin zone for $\mathrm{ZrSiO}$. Purple lines in (c) are nodal lines calculated by first-principles calculations. (d)-(f) are the predicted configurations for node-line degeneracies between the 14th and 15th phonon bands by our algorithm. All the nodal lines carry a quantized $\pi$ Berry phase.

In the last step, we can get the complete configuration for topological degeneracies in BZ by combining the indicators of subgroup \#81 and \#2. First-principles calculations show that there are ten nodal lines including two nodal rings in the BZ, i.e., node-cage degeneracies shown in Fig. 4(c), which perfectly matches the diagnosing results in Fig. 4(f).

Conclusion. We designed a recursive algorithm for diagnosing the complete information of topological degeneracies crossing high-symmetry lines by using the indicators of maximum subgroups. This recursive algorithm can be used in any system with $\mathcal{T}^{2}=+1$ and compensates for the shortcomings of the previous method, which can only be used in systems satisfying compatibility conditions, i.e., no band degeneracies at high-symmetry points or along high-symmetry lines. In two examples for phonon bands, i.e., $\mathrm{In}_{2} \mathrm{Te}$ and $\mathrm{ZrSiO}$, we diagnosed all the topological degeneracies in the Brillouin zone successfully, which verifies the effectiveness of the diagnosing method.

Acknowledgments. T.T.Z. and S.M. acknowledge support from Tokodai Institute for Element Strategy (TIES) funded by MEXT Elements Strategy Initiative to Form Core Research Center. C.F. acknowledges support from the Ministry of Science and Technology of China under Grants No. 2016YFA0302400, No. 2016YFA0300600, and No. 2018YFA0305700; the National Science Foundation of China under Grants No. 11674370, No. 11421092, and No. 11674369; and the Chinese Academy of Sciences under Grants No. XXH13506-202, No. XDB07020100, and No. XDB28000000. L.L. is supported by the National Key R\&D Program of China under Grants No. 2017YFA0303800 and No. 2016YFA0302400 and the NSFC under Project No. 11721404. H.M.W. and Z.F. acknowledge support from the Ministry of Science and Technology of China under Grants No. 2016YFA0300600, No. 2016YFA0302400, and 
No. 2018YFA0305700; the National Natural Science Foundation under Grants No. 11674370, No. 11421092, and No.
11674369; and the Chinese Academy of Sciences under Grants No. XDB28000000 and No. XXH13506-202.
[1] H. C. Po, A. Vishwanath, and H. Watanabe, Symmetry-based indicators of band topology in the 230 space groups, Nat. Commun. 8, 50 (2017).

[2] B. Bradlyn, L. Elcoro, J. Cano, M. G. Vergniory, Z. Wang, C. Felser, M. I. Aroyo, and B. A. Bernevig, Topological quantum chemistry, Nature (London) 547, 298 (2017).

[3] X.-L. Qi and S.-C. Zhang, Topological insulators and superconductors, Rev. Mod. Phys. 83, 1057 (2011).

[4] C. L. Kane and T. C. Lubensky, Topological boundary modes in isostatic lattices, Nat. Phys. 10, 39 (2014).

[5] Z. Song, T. Zhang, Z. Fang, and C. Fang, Quantitative mappings between symmetry and topology in solids, Nat. Commun. 9, 3530 (2018).

[6] Z. Song, T. Zhang, and C. Fang, Diagnosis for Nonmagnetic Topological Semimetals in the Absence of Spin-Orbital Coupling, Phys. Rev. X 8, 031069 (2018).

[7] See Supplemental Material at http://link.aps.org/supplemental/ 10.1103/PhysRevResearch.2.022066 for (i) definition of compatibility condition, (ii) discussions on the algorithm in spinful systems, and (iii) surface states and arcs for the Weyl phonons at $2.58 \mathrm{THz}$.

[8] T. Zhang, Y. Jiang, Z. Song, H. Huang, Y. He, Z. Fang, H. Weng, and C. Fang, Catalogue of topological electronic materials, Nature (London) 566, 475 (2019).

[9] M. G. Vergniory, L. Elcoro, C. Felser, N. Regnault, B. A. Bernevig, and Z. Wang, A complete catalogue of high-quality topological materials, Nature (London) 566, 480 (2019).

[10] F. Tang, H. C. Po, A. Vishwanath, and X. Wan, Comprehensive search for topological materials using symmetry indicators, Nature (London) 566, 486 (2019).

[11] H. Weng, C. Fang, Z. Fang, B. A. Bernevig, and X. Dai, Weyl Semimetal Phase in Noncentrosymmetric TransitionMetal Monophosphides, Phys. Rev. X 5, 011029 (2015).

[12] C. Fang, M. J. Gilbert, X. Dai, and B. A. Bernevig, Multi-Weyl Topological Semimetals Stabilized by Point Group Symmetry, Phys. Rev. Lett. 108, 266802 (2012).

[13] G. Xu, H. Weng, Z. Wang, X. Dai, and Z. Fang, Chern Semimetal and the Quantized Anomalous Hall Effect in $\mathrm{HgCr}_{2} \mathrm{Se}_{4}$, Phys. Rev. Lett. 107, 186806 (2011).

[14] S.-M. Huang, S.-Y. Xu, I. Belopolski, C.-C. Lee, G. Chang, T.-R. Chang, B. Wang, N. Alidoust, G. Bian, and M. Neupane et al., New type of Weyl semimetal with quadratic double Weyl fermions, Proc. Natl. Acad. Sci. USA 113, 1180 (2016).

[15] B. Bradlyn, J. Cano, Z. Wang, M. G. Vergniory, C. Felser, R. J. Cava, and B. A. Bernevig, Beyond Dirac and Weyl fermions: Unconventional quasiparticles in conventional crystals, Science 353, aaf5037 (2016).

[16] Z. Wang, H. Weng, Q. Wu, X. Dai, and Z. Fang, Threedimensional Dirac semimetal and quantum transport in $\mathrm{Cd}_{3} \mathrm{As}_{2}$, Phys. Rev. B 88, 125427 (2013).

[17] Z. Wang, Y. Sun, X.-Q. Chen, C. Franchini, G. Xu, H. Weng, S X. Dai, and Z. Fang, Dirac semimetal and topological phase transitions in $A_{3} \mathrm{Bi}(A=\mathrm{Na}, \mathrm{K}, \mathrm{Rb})$, Phys. Rev. B 85, 195320 (2012).

[18] Z. K. Liu, B. Zhou, Y. Zhang, Z. J. Wang, H. M. Weng, D. Prabhakaran, S.-K. Mo, Z. X. Shen, Z. Fang, X. Dai et al., Discovery of a three-dimensional topological Dirac semimetal, $\mathrm{Na}_{3} \mathrm{Bi}$, Science 343, 864 (2014).

[19] R. M. Geilhufe, S. S. Borysov, A. Bouhon, and A. V. Balatsky, Data mining for three-dimensional organic Dirac materials: Focus on space group 19, Sci. Rep. 7, 7298 (2017).

[20] Q. Xu, Z. Song, S. Nie, H. Weng, Z. Fang, and Xi Dai, Two-dimensional oxide topological insulator with iron-pnictide superconductor LiFeAs structure, Phys. Rev. B 92, 205310 (2015).

[21] C. Fang, H. Weng, Xi Dai, and Z. Fang, Topological nodal line semimetals, Chin. Phys. B 25, 117106 (2016).

[22] B. Lv, H. M. Weng, B. B. Fu, X. P. Wang, H. Miao, J. Ma, P. Richard, X. C. Huang, L. X. Zhao, G. F. Chen et al., Experimental Discovery of Weyl Semimetal TaAs, Phys. Rev. X 5, 031013 (2015).

[23] S.-Y. Xu, I. Belopolski, N. Alidoust, M. Neupane, G. Bian, C. Zhang, R. Sankar, G. Chang, Z. Yuan, C.-C. Lee et al., Discovery of a Weyl Fermion semimetal and topological Fermi arcs, Science 349, 613 (2015).

[24] X. Wan, A. M. Turner, A. Vishwanath, and S. Y. Savrasov, Topological semimetal and Fermi-arc surface states in the electronic structure of pyrochlore iridates, Phys. Rev. B 83, 205101 (2011).

[25] W. Chen, M. Xiao, and C. Chan, Photonic crystals possessing multiple Weyl points and the experimental observation of robust surface states, Nat. Commun. 7, 13038 (2016).

[26] D. T. Son and B. Z. Spivak, Chiral anomaly and classical negative magnetoresistance of Weyl metals, Phys. Rev. B 88, 104412 (2013).

[27] X. Huang, L. Zhao, Y. Long, P. Wang, D. Chen, Zhanhai Yang, H. Liang, M. Xue, H. Weng, Z. Fang et al., Observation of the Chiral-Anomaly-Induced Negative Magnetoresistance in 3D Weyl Semimetal TaAs, Phys. Rev. X 5, 031023 (2015).

[28] J. Xiong, S. K. Kushwaha, T. Liang, J. W. Krizan, M. Hirschberger, W. Wang, R. J. Cava, and N. P. Ong, Evidence for the chiral anomaly in the Dirac semimetal $\mathrm{Na}_{3} \mathrm{Bi}$, Science 350, 413 (2015).

[29] A. A. Zyuzin and A. A. Burkov, Topological response in Weyl semimetals and the chiral anomaly, Phys. Rev. B 86, 115133 (2012).

[30] H. B. Nielsen and M. Ninomiya, The adler-bell-jackiw anomaly and weyl fermions in a crystal, Phys. Lett. B 130, 389 (1983).

[31] L. Lu, J. D. Joannopoulos, and M. Soljačić, Topological photonics, Nat. Photonics 8, 821 (2014).

[32] L. Lu, J. D. Joannopoulos, and M. Soljačić, Topological states in photonic systems, Nat. Phys. 12, 626 (2016).

[33] L. Lu, Z. Wang, D. Ye, L. Ran, L. Fu, J. D. Joannopoulos, and M. Soljačić, Experimental observation of Weyl points, Science 349, 622 (2015). 
[34] T. Zhang, Z. Song, A. Alexandradinata, H. Weng, C. Fang, L. Lu, and Z. Fang, Double-Weyl Phonons in Transition-Metal Monosilicides, Phys. Rev. Lett. 120, 016401 (2018).

[35] H. Miao, T. T. Zhang, L. Wang, D. Meyers, A. H. Said, Y. L. Wang, Y. G. Shi, H. M. Weng, Z. Fang, and M. P. M. Dean, Observation of Double Weyl Phonons in Parity-Breaking FeSi, Phys. Rev. Lett. 121, 035302 (2018).

[36] R. Fleury, A. B. Khanikaev, and A. Alù, Floquet topological insulators for sound, Nat. Commun. 7, 11744 (2016).

[37] S. H. Mousavi, A. B. Khanikaev, and Z. Wang, Topologically protected elastic waves in phononic metamaterials, Nat. Commun. 6, 8682 (2015).

[38] B. Gin-ge Chen, N. Upadhyaya, and V. Vitelli, Nonlinear conduction via solitons in a topological mechanical insulator, Proc. Natl. Acad. Sci. USA 111, 13004 (2014).

[39] R. Süsstrunk and S. D. Huber, Observation of phononic helical edge states in a mechanical topological insulator, Science $\mathbf{3 4 9}$, 47 (2015).

[40] L. M. Nash, D. Kleckner, A. Read, V. Vitelli, A. M. Turner, and W. T. M. Irvine, Topological mechanics of gyroscopic metamaterials, Proc. Natl. Acad. Sci. USA 112, 14495 (2015).

[41] D. Z. Rocklin, B. Gin-ge Chen, M. Falk, V. Vitelli, and T. C. Lubensky, Mechanical Weyl Modes in Topological Maxwell Lattices, Phys. Rev. Lett. 116, 135503 (2016).

[42] Z. Yang, F. Gao, X. Shi, X. Lin, Z. Gao, Y. Chong, and B. Zhang, Topological Acoustics, Phys. Rev. Lett. 114, 114301 (2015).

[43] C. He, X. Ni, H. Ge, X.-C. Sun, Y.-B. Chen, M.-H. Lu, X.-P. Liu, and Y.-F. Chen, Acoustic topological insulator and robust one-way sound transport, Nat. Phys. 12, 1124 (2016).

[44] S. D. Huber, Topological mechanics, Nat. Phys. 12, 621 (2016).

[45] R. Süsstrunk and S. D. Huber, Classification of topological phonons in linear mechanical metamaterials, Proc. Natl. Acad. Sci. USA 113, E4767 (2016).
[46] W.-C. Ji and J.-R. Shi, Topological phonon modes in a twodimensional Wigner crystal, Chin. Phys. Lett. 34, 036301 (2017).

[47] Y. Liu, Y. Xu, S.-C. Zhang, and W. Duan, Model for topological phononics and phonon diode, Phys. Rev. B 96, 064106 (2017).

[48] B. W. Xia, R. Wang, Z. J. Chen, Y. J. Zhao, and H. Xu, Symmetry-Protected Ideal Type-II Weyl Phonons in CdTe, Phys. Rev. Lett. 123, 065501 (2019).

[49] K. Li, C. Li, J. Hu, Y. Li, and C. Fang, Dirac and Nodal Line Magnons in Three-Dimensional Antiferromagnets, Phys. Rev. Lett. 119, 247202 (2017).

[50] W. Yao, C. Li, L. Wang, S. Xue, Y. Dan, K. Iida, K. Kamazawa, K. Li, C. Fang, and Y. Li, Topological spin excitations in a threedimensional antiferromagnet, Nat. Phys. 14, 1011 (2018).

[51] A. P. Schnyder, S. Ryu, A. Furusaki, and A. W. W. Ludwig, Classification of topological insulators and superconductors in three spatial dimensions, Phys. Rev. B 78, 195125 (2008).

[52] C.-K. Chiu, J. C. Y. Teo, A. P. Schnyder, and S. Ryu, Classification of topological quantum matter with symmetries, Rev. Mod. Phys. 88, 035005 (2016).

[53] S. Ryu, A. P. Schnyder, A. Furusaki, and A. W. W. Ludwig, Topological insulators and superconductors: Tenfold way and dimensional hierarchy, New J. Phys. 12, 065010 (2010).

[54] G. A. Gamal, A. T. Nagat, M. M. Nassary, and A. M. AbouAlwafa, Some physical properties of $\mathrm{Ga}_{2} \mathrm{Se}$ single crystals, Cryst. Res. Technol. 31, 359 (1996).

[55] X. Gonze and C. Lee, Dynamical matrices, born effective charges, dielectric permittivity tensors, and interatomic force constants from density-functional perturbation theory, Phys. Rev. B 55, 10355 (1997).

[56] G. Kresse and J. Furthmüller, Efficient iterative schemes for ab initio total-energy calculations using a plane-wave basis set, Phys. Rev. B 54, 11169 (1996).

[57] H. Onken, K. Vierheilig, and H. Hahn, Über silicid-und germanidchalkogenide des zirkons und hafniums, Z. Anorg. Allg. Chem. 333, 267 (1964). 